\title{
A Fuzzy Control System for Improving Learning
}

\author{
Carlos Lara-Alvarez ${ }^{1}$, Oyuki Fuentes ${ }^{2}$, Hugo Mitre-Hernandez ${ }^{2}$, \\ Juan J. Flores ${ }^{3}$, Luis Lopez ${ }^{2}$ \\ ${ }^{1}$ CONACYT Research Fellow, Centro de Investigación en Matemáticas, \\ Mexico \\ ${ }^{2}$ Laboratorio de Interacción Humano-Computador, \\ Centro de Investigación en Matemáticas, \\ Mexico \\ ${ }^{3}$ Facultad de Ingeniería Eléctrica, Universidad Michoacana. \\ Morelia, Mexico \\ \{carlos.lara, maria.fuentes, hmitre, luis.lopez\}@cimat.mx juanf@umich.mx
}

\begin{abstract}
It has been shown that the emotional state of students has an important relationship with learning; for instance, engaged concentration is positively correlated with learning. This paper proposes the Fuzzy Control for Improving Learning (FCIL), the proposed technique induces emotions in the player for supporting the learning process. The fuzzy system analyzes the players' emotional state for controlling the aesthetic content of an educational video game. A total of 13 subjects played a video game designed to practice basic math skills; for each trial, a student plays two times in a row the same game but each time the game was controlled by one of two approaches: FCIL or random. Results show that when the proposed approach is used, participants showed more engagement, i.e., they were in pleasant-high states more frequently. The proposed approach is a promising alternative for improving the learning effectiveness through detection and stimulation of positive emotions.
\end{abstract}

Keywords: Fuzzy control, educational video games, emotion induction.

\section{Introduction}

An educational videogame is a computer game that induces user engagement while promoting cognitive learning and social skills. In general, a successful educational game requires that its mechanics (game components), dynamics (behavior as responses of the players inputs), and aesthetics (environment shapes, animations, sound, etc.) fulfill the expectations of the target population. Each player has specific characteristics, e.g., preferences, abilities, emotions, and the game must adjust its dynamics and aesthetics accordingly.

In general, there are two approaches to adjust the game content: Dynamic Difficulty Adjustment (DDA) methods, aka dynamic game balancing, automatically change parameters, scenarios, and behaviors in a video game in real-time, based on the player's 
ability; on the other hand, Emotionally Adaptive (EA) methods consider that by maintaining a high level of engagement, the game experience could lead to psychological benefits, such as a sense of efficacy and power over one's environment, as well as improvements in learning.

The main contribution of this paper is a fuzzy-logic-based method for changing the aesthetics of an educative video game. The aim of this approach is to select the aesthetic content for promoting learning. We believe that some multimedia content can: (i) alleviate some negative emotional states that are common in the constructive learning process (e.g., frustration), (ii) activate students that are in states that prevent learning (e.g., boredom or tiredness), and (iii) keep students in states that favor learning (e.g., happiness). The fuzzy rules were designed to consider many of these cases, it is important to avoid unlearning states for long periods; e.g., it is worthwhile to use unpleasant sounds to stimulate a bored student that cannot be activated by using harder difficulty level nor using pleasant sounds.

The rest of this paper, is organized as follows: section 2 reviews alternative approaches proposed to adjust the content of an educational video game, section 2 introduces the proposed approach, section 4 describes the empirical study, section 5 presents and discusses the results, and section 6 provides some conclusions together with perspectives for future work.

\section{Related Work}

There are many studies that focus on providing instructional objects at the correct level of difficulty for the student. A common approach consists of planning the curriculum sequence, i.e. providing the student with the most suitable sequence of knowledge units and learning tasks (examples, questions, problems, etc.) [3]. This approach solves the problem of finding an optimal path through the learning material.

Few studies have investigated how to improve the game--based learning performance by adapting the instructional material according to the learner's current developmental and individual particularities; e.g., the way of thinking, feeling, behaving, and relating to others; for instance, some approaches [7, 5] identify the learning style for providing personalized learning materials - learning styles group common ways that people learn [6].

Emotionally Adaptive methods require knowing the emotional state to take decisions. These decisions can only consider specific emotional feature; e.g., the mental work load [17] the anxiety level [15, 10] or the arousal [13].

Emotions can be defined as biologically based action dispositions that have an important role in the determination of behavior [9]. Emotional response can be measured by different approaches: affective reports, overt behavioral, and physiological reactivity [8]. Affective reports require that an observer (someone or the user himself) determines the user's emotional state; many instruments aiming to support the evaluation process have been proposed - e.g., adjective checklists, physiological techniques, photo decks [12]. In this paper, a simplified version of the Self-Assessment Manikin (SAM) [12] was used to assess the user's emotional state. SAM is a non-verbal pictorial assessment technique that uses a graphic figure depicting values along each of 
three dimensions (pleasure, arousal, and dominance). The simplified version used in this paper only uses two dimensions (pleasure and arousal).

A closed related work is that of Liu et al. [10] where the player's physiological signals are analyzed to infer his or her probable anxiety level and the game difficulty level is automatically adjusted in real time as a function of the player's affective state. They use a finite state machine to model affection-based dynamic difficulty adjustment; but, their approach does not adjust the game contents.

\section{Fuzzy Control}

The inputs for the fuzzy control are: $(\underline{V}, \underline{A})$, the current player's emotional state; and $\left(V_{k-1}, A_{k-1}\right)$, the nominal emotion induced by the aesthetics used in the previous stage, where $V$ is the valence and $A$ is the arousal. The fuzzy control output is the aesthetic $\left(V_{k-1}, A_{k-1}\right)$ for stage $k$.

\subsection{Membership Functions}

We need to define the linguistic terms that input/output variables can take on. Along with each linguistic term, we need to define their membership functions. Although most of the work that deals with fuzzy inference and control, uses an odd number of linguistic terms, that is not a requirement. As shown in Fig. 1, the variables we use in performing fuzzy inference are defined with 2, 3, 4, or 5 linguistic terms; the number of them and their labels were taken from the application domain [14]. Another important design decision about the fuzzy inference system is the shape of the membership functions for the linguistic terms of the involved variables. Gaussian functions were chosen to represent fuzzy set membership.

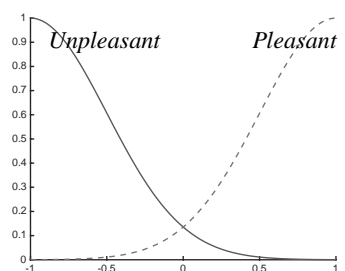

(a)

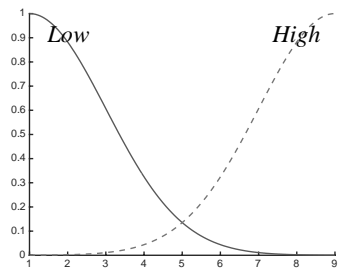

(d)

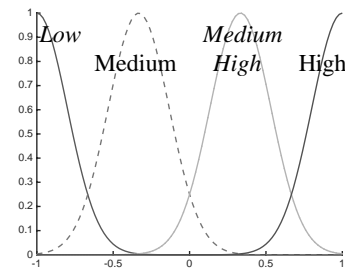

(b)

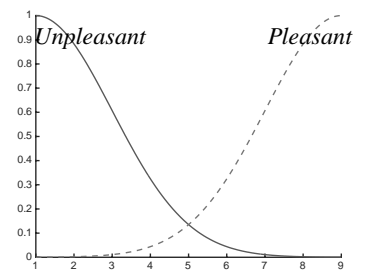

(e)

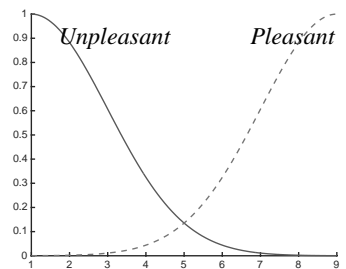

(c)

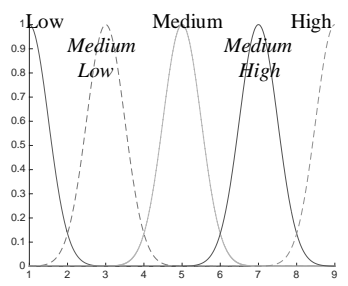

(f)

Fig. 1. Membership functions. (a) $\underline{V} \quad$ (b) $\underline{A}$ (c) $V_{k-1} \quad$ (d) $A_{k-1} \quad$ (e) $V_{k} \quad$ (f) $A_{k}$. 


\subsection{Fuzzy Rules}

To induce emotions in the player, the current implementation only change sounds, but the results can be generalized to other aesthetics. To induce emotions, the IADS-2 International Affective Digitized Sounds [2] data set was used. The IADS was developed to provide a set of normative emotional stimuli for experimental investigations of emotion and attention.

Table 1 Fuzzy Rules for the next stage aesthetics by considering the player emotional state and the previous aesthetics used. The notation (Valence, Arousal Level) represents the state. The subscript in each cell indicates the rule number. Pleasant emotions are represented as + , and Unpleasant as -.

\begin{tabular}{|c|c|c|c|c|}
\hline & \multicolumn{3}{|c|}{ Previous Aesthetics } \\
\hline & & $(+$, Low $)$ & (+, High) & (-, High) \\
\hline \multirow{8}{*}{ 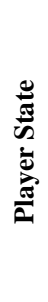 } & $(+$, Low $)$ & $\mathrm{R}_{1}:$ (+, Very High) & $\mathrm{R}_{2}:(-$, High $)$ & $\mathrm{R}_{3}:(+$, Very High $)$ \\
\hline & (+, Medium) & $\mathrm{R}_{4}:(+$, High $)$ & $\mathrm{R}_{5}:(+, \mathrm{High})$ & $\mathrm{R}_{6}:(+$, High $)$ \\
\hline & (+, Medium-high) & $\mathrm{R}_{7}:(+$, Medium $)$ & $\mathrm{R}_{8}:(+$, Medium $)$ & $\mathrm{R}_{9}:(-$, Medium $)$ \\
\hline & $(+$, High $)$ & $\mathrm{R}_{10}:(+$, Medium $)$ & $\mathrm{R}_{11}:(+$, Medium $)$ & $\mathrm{R}_{12}:(-$, Medium $)$ \\
\hline & (-, Low) & $\mathrm{R}_{13}:(+$, Very High $)$ & $\mathrm{R}_{14}:(-, \mathrm{High})$ & $\mathrm{R}_{15}:$ (+, Very High) \\
\hline & (-, Medium) & $\mathrm{R}_{16}:(+$, High $)$ & $\mathrm{R}_{17}:(-$, High $)$ & $\mathrm{R}_{18}:(+$, High $)$ \\
\hline & (-, Medium-high) & $\mathrm{R}_{19}:(+$, Medium Low $)$ & $\mathrm{R}_{20}:(+$, Medium Low $)$ & $\mathrm{R}_{21}:(+$, Medium Low $)$ \\
\hline & (-, Medium-high) & $\mathrm{R}_{22}:(+$, Low $)$ & $\mathrm{R}_{23}:(+$, Low $)$ & $\mathrm{R}_{24}:(+$, Low $)$ \\
\hline
\end{tabular}

The fuzzy rules for aesthetics, shown in Table 1, were selected by applying the following theories and facts:

1. Russell [16] asserts that the emotional state is a biological product of evolution and therefore it likely has a function. Russell suggests a general principle of congruence: pleasant states facilitate attention to positive material (and viceversa).

2. Pleasant-high emotions are associated to engaged concentration, which is positively correlated to learning [16] hence, each rule of $\left\{R_{1}, R_{3} \ldots R_{6}, R_{13}, R_{15}\right.$, $\left.R_{16}, R_{18}\right\}$ selects aesthetics that induce pleasant-high emotions. The rules in this set aim to activate students.

3. Unpleasant-high emotions (e.g., frustration or confusion) may be natural and unavoidable when learning a difficult material [1] such is the case of conditions required for rules $\left\{R_{19} \ldots R_{24}\right\}$. Aiming to reduce the arousal, these rules apply aesthetics that evoke pleasant-low emotions (e.g., calm).

4. Baker et al. [1] suggest that boredom must be detected and quickly managed. Boredom conditions are in rules $\left\{R_{13} \ldots R_{18}\right\}$; hence, these rules suggest arousing aesthetics aiming to elevate the student arousal.

5. For the same reasons stated in the preceding point, the proposed rules avoid inducing unpleasant-low emotions; furthermore, these aesthetics are never used and Table 1 does not have a column labeled as '(-, Low)'. 


\subsection{Fuzzification and Defuzzification}

The fuzzification transforms the inputs into fuzzy sets in such a way that they can be used by the fuzzy system; for this aim, a simple singleton fuzzifier was used. A number of deffuzzification strategies exist, each provides a means to choose a single output based on the implied fuzzy sets. A typical Center Of Gravity (COG) strategy was used [14].

\section{Materials and Methods}

This study investigates the pertinence of the proposed method in terms of players' enjoyment, and performance. The following sections describe the video game, participants and metrics used in this study.

\subsection{Videogame}

A game to practice basic math skills was designed for this study. Two versions of this game were used for the test, they have the same mechanics and dynamics - e.g., both have the same game elements, states, and rules for selecting the difficulty - but different aesthetics, as explained below.

Dynamics. Each stage is composed of two scenes that interact with the player. In the first scene (Fig 2a), a character throws 10 arithmetic operations of two numeric quantities. For each operation, the player must choose the right answer by moving the falling object (using left and right keys); the fall can also be speeded up (using the down key). To obtain points, the falling object (the sum) must be introduced into the correct container (the answer) before it reaches the bottom of the screen. The game plays the selected sound each time a new sum operation is shown. The second scene (Fig 2b) is aimed to obtain the user's emotional state; the user introduces his/her emotional state by using a simplified SAM version [12] that only has two sliders (arousal/valence).

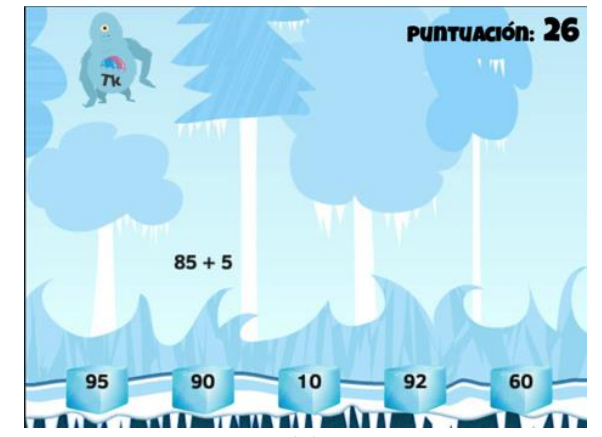

(a)

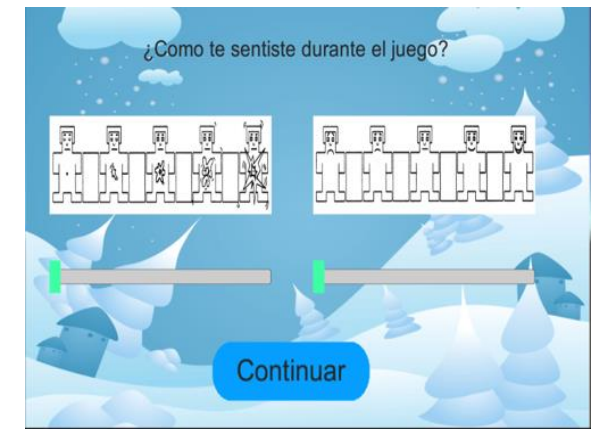

(b)

Fig. 2. Game Dynamics: (a) The playing scene, the user must introduce the falling object into the correct container to earn points, (b) SAM scene, the self-report is used to introduce arousal and activation values. 
Difficulty Level. For testing purposes, games use a simple linear DDA algorithm to select the next difficulty level. The change factor is calculated as

$$
F=S+0.5
$$

where $S$ is the Score Ratio obtained in the previous stage. The new difficulty level, $D_{k}$ is calculated as

$$
D_{k}=F \cdot D_{k-1} \text {. }
$$

where $D_{k-1}$ is the difficulty of the previous stage.

Aesthetics. The two games differ in the strategy employed for generating the next scene content (aesthetic):

Game A. The aesthetics (sound) is selected randomly from the database.

Game B. It uses the FCIL approach proposed in this paper. The aesthetic selector chooses the closest sound to the suggested. For both games, a random content is generated at the beginning and an initial difficulty is selected.

\subsection{Participants and Procedure}

A total of 13 participants in secondary school from the "Colegio del Centro" Secondary School in Zacatecas, Mexico participated in this study. Participants played two consecutive 10-minute play sessions separated by a 5-minute resting period. Each participant played 'Game A' for one session and 'Game B' for the other - the order of playing these sessions was assigned randomly. In total, six participants played first the 'Game A' and then the 'Game B' (and the other seven participants first played the 'Game B').

\subsection{Metrics}

For each participant/play session the following metrics were obtained:

Percentage of stages by emotional state. This metric is calculated as:

$$
e=\frac{{ }_{p} p_{e}}{{ }_{p} p_{t}} 100,
$$

where $p_{e}$, is the number of stages that participant $p$ exhibited emotion $e$ and, $p_{t}$ is the number of stages completed by participant $p$ in the play session.

Emotional Transitions. Let $e, e^{\prime}$ be two emotional states, the percentage of transitions between $e$ and $e^{\prime}$ is:

$$
\operatorname{tran}\left(e, e^{\prime}\right)=\frac{\sum_{\forall p} p_{e \mapsto e^{\prime}}}{\sum_{\forall p} p_{e}},
$$


where $p_{e \mapsto e^{\prime}}$ is the number of times that participant $p$ exhibited the emotion $e$ immediately followed by emotion $e^{\prime}$. Note that

$$
\operatorname{tran}\left(e, e_{i}\right)=1
$$

Last Score. To compare the performance of participants, video games save data log of

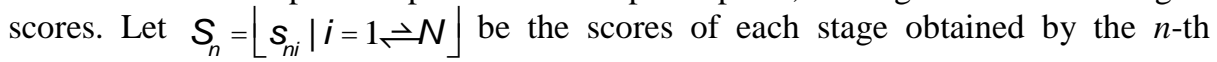
participant, where $s_{n i}$ is the score at the end of the $i$-th completed stage. The last score is

$$
\operatorname{last}\left(S_{n}\right)=s_{n} N
$$

\subsection{Statistical Analysis}

Data are represented as mean \pm S.D. and the significance was assessed by Student's ttest for paired data. Differences between values were considered significant when $\mathrm{p}<0.05$.

\section{$5 \quad$ Results}

The overall comparison of the proportion of stages by emotional group is shown in Fig. 3. The proportion of pleasant-high states is higher for FCIL (55.21\%) than for random selection $(40.95 \%)$. Besides, the overall proportion of unpleasant-high states is lower for FCIL (18.75\%) than for random selection $(20.95 \%)$.

Table 2. Comparison of emotional state transitions. As shown in bold, transitions to

\begin{tabular}{|c|c|c|c|c|c|c|c|c|c|c|}
\hline & \multicolumn{4}{|c|}{ Random } & \multicolumn{4}{|c|}{ FCIL } \\
\hline & & & \multicolumn{4}{|c|}{$(\text { Emotional state })_{\mathrm{k}+1}$} & \multicolumn{4}{|c|}{$(\text { Emotional state })_{k+1}$} \\
\hline & & & \multicolumn{2}{|c|}{ Pleasant } & \multicolumn{2}{|c|}{ Unpleasant } & \multicolumn{2}{|c|}{ Pleasant } & \multicolumn{2}{|c|}{ Unpleasant } \\
\hline & & & Low & High & Low & High & Low & High & Low & High \\
\hline \multirow{4}{*}{ 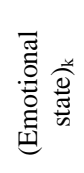 } & \multirow{2}{*}{ Pleasant } & Low & 0.633 & 0.200 & 0.100 & 0.067 & 0.278 & 0.500 & 0.056 & 0.167 \\
\hline & & High & 0.128 & 0.564 & 0.026 & 0.282 & 0.130 & 0.696 & 0.022 & 0.152 \\
\hline & \multirow{2}{*}{ Unpleasant } & Low & 0.600 & 0.200 & 0.200 & 0.000 & 0.167 & 0.500 & 0.167 & 0.167 \\
\hline & & High & 0.056 & 0.444 & 0.056 & 0.444 & 0.000 & 0.308 & 0.231 & 0.462 \\
\hline
\end{tabular}
pleasant-high are higher for the proposed approach than for random selection.

As shown in Table 2 transitions to pleasant-high are higher for the proposed approach than for random selection (except for unpleasant-high states). It means that participants change to pleasant states more easily by using the proposed approach. Furthermore, unpleasant-high emotional states not always require remediation [11] since such states (e.g., frustration or confusion) are related to learning and are linked to learning gains [1].

Finally, there was not a statistical difference in the last score for FCIL and random approaches, $\mathrm{t}(13)=-1.30, \mathrm{p}=0.217$. It means that results shown in Fig. 3 and Table 2 
was not driven by the difficulty level; hence, the aesthetics are playing a crucial role to change the emotional state of the player.

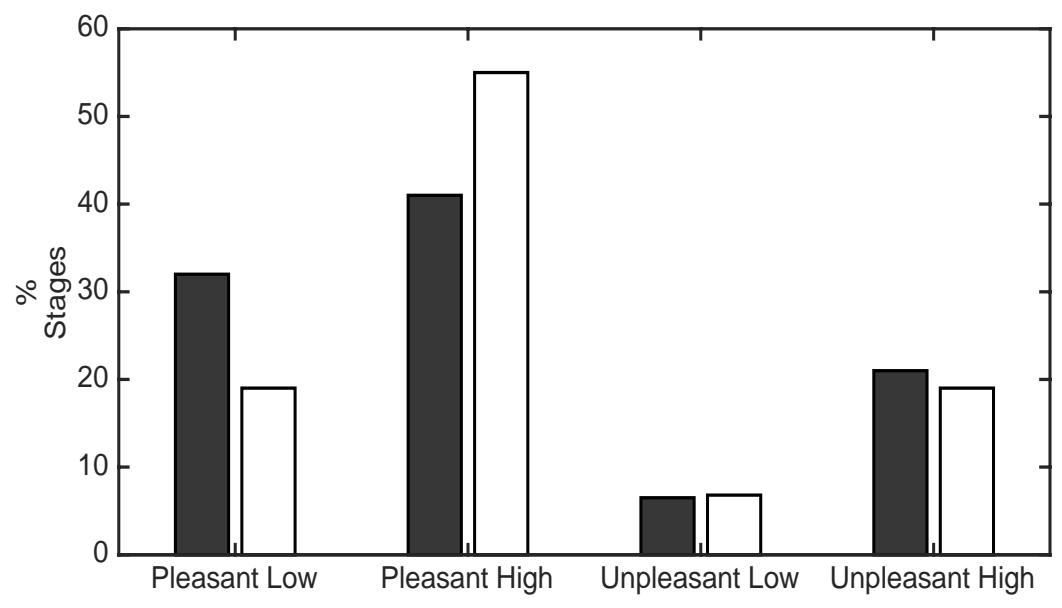

Fig. 3. Overall comparison of percentage of stages by emotional state of the player (dark random selection, white - proposed approach).

\section{Conclusions and Further Work}

The proposed technique induces emotions in the player for supporting the learning process. The overall results show that in comparison to random content generation, the proposed approach increases the proportion of stages were the students experience pleasant-high emotions and reduces their Unpleasant-low stages. The unpleasanttlow is considered an unlearning state; hence it must be avoided because it can make it difficult for students to complete some tasks. Currently, we are working on a systemic approach that controls the difficulty, the mechanics, dynamics and aesthetics of learning materials. Introducing a difficulty control into the proposed FCIL could help students to smoothly reach their "flow zone" [4] and once reached, it can help to keep students in flow. Besides, we are working on replacing the self-reporting by a physiological assessment.

Acknowledgments. We are in debt to the staff members and students of the secondary school "Juan Bosco" from Zacatecas, Mexico. This research was funded by the National Council of Science and Technology of Mexico (CONACyT) through the project CATEDRAS-3163.

\section{References}

1. Baker, R. S., D’Mello, S. K., Rodrigo, M. T., Graesser, A. C.: Better to be frustrated than bored: The incidence, persistence, and impact of learners cognitive-affective states during 
interactions with three different computer-based learning environments. International Journal of Human-Computer Studies, 68(4), pp. 223-241 (2010)

2. Bradley, M. M., Lang, P. J.: The international affective digitized sounds (iads-2): Affective ratings of sounds and instruction manual. University of Florida, Gainesville, FL, Tech. Rep. B-3 (2007)

3. Brusilovsky, P.: Adaptive and intelligent technologies for web-based education. KI, 13(4), pp. 19-25(1999)

4. Csikszentmihalyi, M.: Flow: The psychology of optimal experience. Harper Perennial Modern Classics (2008)

5. Dascalu, M. I., Bodea, C. N., Moldoveanu, A., Mohora, A., Lytras, M., de Pablos, P. O.: A recommender agent based on learning styles for better virtual collaborative learning experiences. Computers in Human Behavior, 45, pp. 243-253 (2015)

6. Felder, R. M., Silverman, L. K.: Learning and teaching styles in engineering education. Engineering education, 78(7), pp. 674-681 (1988)

7. Hung, Y. H., Chang, R. I., Lin, C. F.: Hybrid learning style identification and developing adaptive problem-solving learning activities. Computers in Human Behavior, 55, pp. 552$561(2016)$

8. Lang, P. J.: The mechanics of desensitization and the laboratory study of human fear. Behavior therapy: Appraisal and status. New York, McGraw-Hill, pp. 160-191 (1969)

9. Lang, P. J.: The emotion probe: studies of motivation and attention. American psychologist 50(5), pp. 372 (1995)

10. Liu, C., Agrawal, P., Sarkar, N., Chen, S.: Dynamic difficulty adjustment in computer games through real-time anxiety-based affective feedback. International Journal of HumanComputer Interaction, 25(6), pp. 506-529 (2009)

11. Mentis, H. M.: Memory of frustrating experiences. Information and Emotion: The Emergent Affective Paradigm in Information Behavior Research and Theory, Eds. Diane Nahl and Dania Bilal, pp. 197-210 (2007)

12. Morris, J. D.: Observations: Sam: the self-assessment manikin; an efficient cross-cultural measurement of emotional response. Journal of advertising research, 35(6), pp. 63-68 (1995)

13. Parnandi, A., Gutierrez-Osuna, R.: A comparative study of game mechanics and control laws for an adaptive physiological game. Journal on Multimodal User Interfaces, 9(1), pp. 31-42 (2015)

14. Passino, K. M., Yurkovich, S., Reinfrank, M.: Fuzzy control. Addison Wesley, 20 (1998)

15. Rani, P., Sarkar, N., Liu, C.: Maintaining optimal challenge in computer games through real-time physiological feedback. In: Proceedings of the 11th international conference on human computer interaction, 58, pp. 184-192 (2005)

16. Russell, J. A.: Core affect and the psychological construction of emotion. Psychological review, 110(1), pp. 145 (2003)

17. Takahashi, M., Tsuyoshi, A., Kuba, O., Yoshikawa, H.: Experimental study toward mutual adaptive interface. In: Proceedings of the 3rd IEEE International Workshop on Robot and Human Communication (RO-MAN'94) Nagoya, IEEE, pp. 271-276 (1994) 